\title{
Aortic Valve Stenosis Increases Helical Flow and Flow Complexity: A Study of Intra- operative Cardiac Vector Flow Imaging
}

Hansen, Kristoffer Lindskov; Møller-Sørensen, Hasse; Kjaergaard, Jesper; Jensen, Maiken Brit; Jensen, Jørgen Arendt; Nielsen, Michael Bachmann

Published in:

Ultrasound in Medicine and Biology

Link to article, DOI:

10.1016/j.ultrasmedbio.2017.03.018

Publication date:

2017

Document Version

Peer reviewed version

Link back to DTU Orbit

Citation $(A P A)$ :

Hansen, K. L., Møller-Sørensen, H., Kjaergaard, J., Jensen, M. B., Jensen, J. A., \& Nielsen, M. B. (2017). Aortic Valve Stenosis Increases Helical Flow and Flow Complexity: A Study of Intra-operative Cardiac Vector Flow Imaging. Ultrasound in Medicine and Biology, 43(8), 1607-1617.

https://doi.org/10.1016/j.ultrasmedbio.2017.03.018

\section{General rights}

Copyright and moral rights for the publications made accessible in the public portal are retained by the authors and/or other copyright owners and it is a condition of accessing publications that users recognise and abide by the legal requirements associated with these rights.

- Users may download and print one copy of any publication from the public portal for the purpose of private study or research.

- You may not further distribute the material or use it for any profit-making activity or commercial gain

- You may freely distribute the URL identifying the publication in the public portal 


\title{
AORTIC VALVE STENOSIS INCREASES HELICAL FLOW AND FLOW COMPLEXITY: A STUDY OF INTRA-OPERATIVE CARDIAC VECTOR FLOW IMAGING
}

\author{
Kristoffer Lindskov Hansen, ${ }^{*}$ Hasse Møller-Sørensen, ${ }^{\dagger}$ Jesper KJaERgaArd, $^{\dagger}$

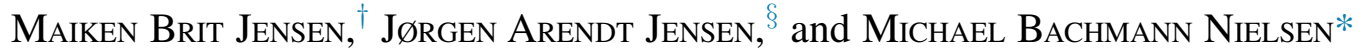 \\ * Radiology Department, Rigshospitalet, Denmark; ${ }^{\dagger}$ Cardiothoracic Anesthesiology Department, Rigshospitalet, Denmark; \\ ${ }^{\ddagger}$ Cardiology Department, Rigshospitalet, Denmark; and ${ }^{\S}$ Center for Fast Ultrasound Imaging, DTU Elektro, Technical \\ University of Denmark, Denmark
}

(Received 15 November 2016; revised 21 March 2017; in final form 27 March 2017)

\begin{abstract}
Aortic valve stenosis alters blood flow in the ascending aorta. Using intra-operative vector flow imaging on the ascending aorta, secondary helical flow during peak systole and diastole, as well as flow complexity of primary flow during systole, were investigated in patients with normal, stenotic and replaced aortic valves. Peak systolic helical flow, diastolic helical flow and flow complexity during systole differed between the groups $(p<0.0001)$, and correlated to peak systolic velocity $(R=0.94,0.87$ and 0.88 , respectively $)$. The study indicates that aortic valve stenosis increases helical flow and flow complexity, which are measurable with vector flow imaging. For assessment of aortic stenosis and optimization of valve surgery, vector flow imaging may be useful. (E-mail: lindskov@gmail.com) (c) 2017 World Federation for Ultrasound in Medicine \& Biology.
\end{abstract}

Key Words: Vector flow imaging, Ascending aorta, Aortic valve stenosis, Helical flow, Flow complexity.

\section{INTRODUCTION}

Helical flow is recognized as a normal flow pattern in the vascular system and is perceived as a secondary rotational motion of the blood normal to the mainstream of the primary flow (Bogren and Buonocore 1999; Liu et al. 2015; Markl et al. 2004). It has been observed in numerous vessel geometries, for example, in the heart (Elbaz et al. 2014), the aorta (Hansen et al. 2016a; Kilner et al. 1993), the carotid and femoral arteries (Pedersen et al. 2011; Sarrami-Foroushani et al. 2015) and the veins of the lower extremities (Lurie and Kistner 2013), and has been explained by several theories. It has been suggested that helical flow stabilizes flow, preserves energy and protects against atherosclerosis (Kilner et al. 1993; Liu et al. 2009b; Morbiducci et al. 2011). Studies have indicated that the helical flow pattern facilitates blood flow transport to vessel branches, thereby optimizing organ perfusion and oxygen delivery, and is associated with lower lipid

Address correspondence to: Kristoffer Lindskov Hansen, Radiology Department, Rigshospitalet, Blegdamsvej 9, 2100 Copenhagen, Denmark. E-mail: lindskov@gmail.com concentration along the inner vessel wall (Liu et al. 2009a, 2009b; Stonebridge and Brophy 1991).

Disease in the cardiovascular system is often accompanied by altered flow patterns; for example, aortic valve stenosis can be assessed with echocardiography as an increase in systolic velocities, pressure gradients and flow complexity (Nishimura et al. 2014; Simpson et al. 1988), where flow complexity can be evaluated by spectral broadening, power intensity and mosaic patterns using spectral, power and color Doppler ultrasound (US), respectively (Cloutier et al. 1995; Hutchison et al. 1996; Stringer et al. 1989). Helical flow is also affected by cardiovascular disease. Studies of blood flow in the ascending aorta in patients with bicuspid aortic valve and aortic valve stenosis have reported increased helical flow, which has been linked to development of aortic aneurysm (Hope et al. 2010; Meierhofer et al. 2013; von Knobelsdorff-Brenkenhoff et al. 2016).

The majority of studies of helical blood flow have been conducted with magnetic resonance imaging (MRI) and, to a lesser extent, conventional Doppler US (Bogren and Buonocore 1999; Frazin et al. 1996; Hope et al. 2010; Kilner et al. 1993; Koh et al. 2001; Liu et al. 2015; Markl et al. 2004; Meierhofer et al. 2013; 
Sarrami-Foroushani et al. 2015; Segadal and Matre 1987; von Knobelsdorff-Brenkenhoff et al. 2016; von Spiczak et al. 2015). However, recent studies have indicated that vector flow imaging (VFI) using US also is a useful modality for helical flow imaging (Hansen et al. 2015, 2016a, 2016b). A study of the helical flow in the ascending aorta during late systole and diastole in patients with normal or stenotic aortic valves did not find any correlation to aortic valve stenosis (Hansen et al. 2016b). It was, however, indicated that there is a short rapid vortical motion in the ascending aorta during peak systole in some patients (Hansen et al. 2016a, 2016b). A measure of flow complexity, where the averaged flow angle is found, has likewise been introduced recently with VFI (Pedersen et al. 2014). Studies of flow in the carotid artery and the ascending aorta have indicated that the measure can distinguish between complex and laminar flow (Hansen et al. 2015, 2016b; Pedersen et al. 2014).

The primary aim of this study was to examine the influence of aortic valve stenosis on secondary helical blood flow in the ascending aorta during peak systole and diastole by comparing blood flow in 10 patients with normal aortic valves to that of 10 patients with aortic valve stenosis before and after valve replacement. Also, the flow complexity of the primary blood flow during systole was estimated with VFI and correlated to peak systolic velocities. The hypothesis was that helical flow and flow complexity in the ascending aorta, when estimated with VFI, can be correlated to aortic valve stenosis in a study of patients with normal, stenotic and replaced aortic valves.

\section{METHODS}

After approval by the local ethics committee (No. $\mathrm{H}-16024707), 20$ patients entered the study after giving written informed consent. The 10 patients in group I (6 males, 4 females, mean age: $63.9 \mathrm{y}$, range: $53-78$ y) had no history of valvular disease and were scheduled for coronary bypass surgery, and the 10 patients in group II ( 9 males, 1 females, mean age: $70.7 \mathrm{y}$, range: $62-80 \mathrm{y}$ ) had aortic valve stenosis and were scheduled for valve replacement with a biologic valve prosthesis.

Blood flow in the ascending aorta was measured with epi-aortic VFI scans in long-axis (LAX) and shortaxis (SAX) views after standard sternotomy and opening of the pericardium, but before cannulation for cardiopulmonary bypass. For the patients with aortic valve stenosis in group II, the epi-aortic VFI scans were repeated after implantation of the biologic prosthetic valve, weaning of bypass and decannulation. Thus, patient group II was scanned twice, over a stenotic aortic valve (group IIa) and over a replaced aortic valve (group IIb). Standard spectral Doppler measurements of blood flow in the ascending aorta were obtained with transesophageal echocardiography (TEE) before each VFI scan in the LAX view.

\section{Vector flow imaging}

The VFI method transverse oscillation, proposed by Jensen and Munk (1998), is an angle-independent vector velocity method, which estimates both the axial and transverse velocity components from each received echo using conventional Doppler pulse emission. The axial velocity component $v_{z}$ is found as in conventional Doppler US with a conventional bell-shaped apodization function in receive, whereas the transverse velocity component $v_{x}$ is found by changing the apodization function in receive to resemble a two-point source and with the use of a special estimator (Fig. 1) (Jensen 2001). Combining the axial and the transverse velocity components for each point within a region of interest (ROI) provides a 2-D vector velocity map of angleindependent blood velocities in the measurement plane. VFI is described in detail in previous articles (Jensen 2001; Jensen and Munk 1998; Udesen and Jensen 2006).

A conventional US scanner (ProFocus 2202 UltraView, BK Medical, Herlev, Denmark) with a linear transducer (8670, BK Medical, Herlev, Denmark) under sterile settings was used to record the epi-aortic scan sequences. Sterile saline was poured into the mediastinal cavity before each epi-aortic scan to enhance the acoustic transmission from the probe to the aortic surface (American Society of Anesthesiologists and Society of Cardiovascular Anesthesiologists Task Force on Transesophageal Echocardiography 2010).

The ascending aorta was scanned in LAX and SAX views $1-2 \mathrm{~cm}$ downstream of the aortic valve for each patient. In SAX view, the secondary helical motion was recorded. To capture both the slow and fast rotational motion of the blood in the diastolic and systolic phases, the blood flow in the ascending aorta in SAX view was measured with both a low and a high pulse repetition frequency (PRF). In LAX view, each patient was only scanned with a high PRF to capture the blood flow during systole for assessment of flow complexity.

For each scan, the color box was adjusted to cover the lumen, in either the longitudinal or transverse direction, and depth setting, gain and wall filtering were adjusted for vector velocity estimation. The averaged applied parameter setting for each patient group is provided in Table 1. For all measurements, the center frequency for $\mathrm{B}$-mode imaging was $9 \mathrm{MHz}$, and that for VFI, $5 \mathrm{MHz}$.

The temporal resolution of the VFI estimation was 16 frames/s, and the maximum scan depth was approximately $5 \mathrm{~cm}$ because of the transducer setup available. 
a
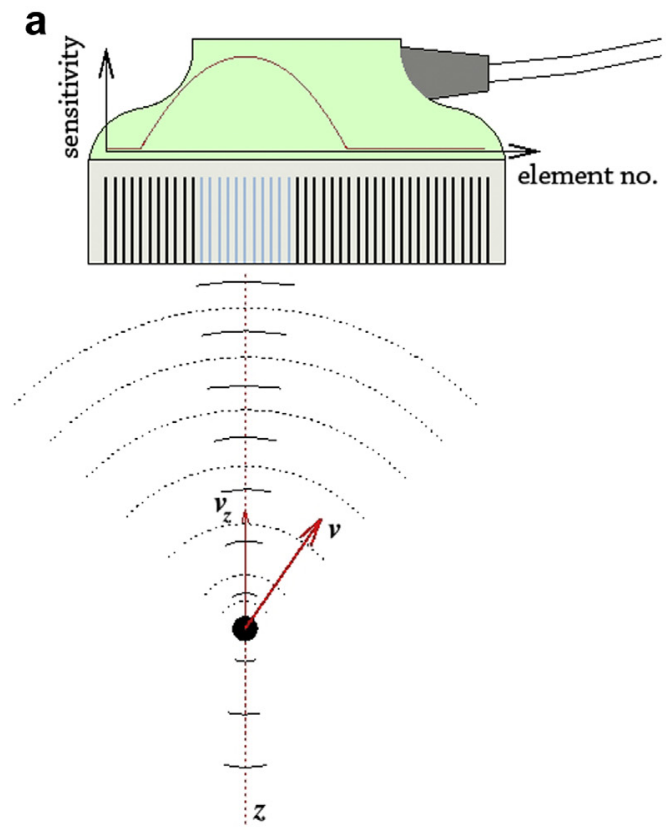

b
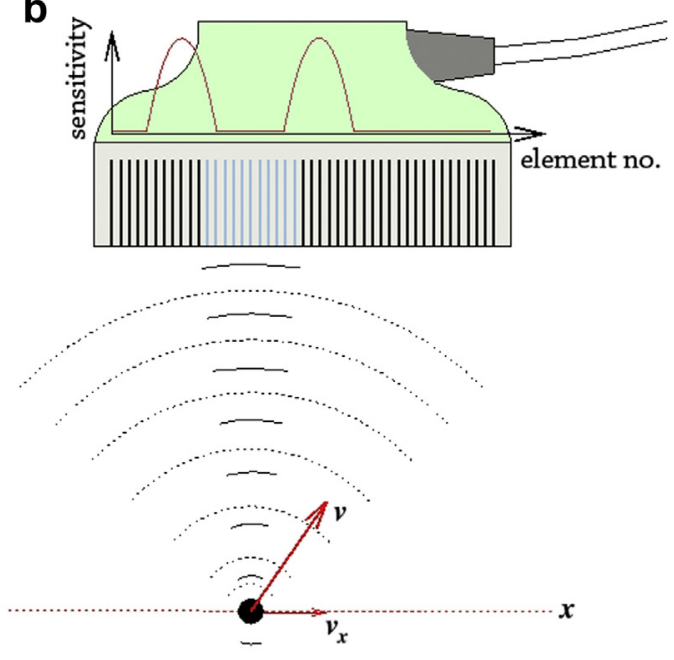

Fig. 1. Echo received from a scatterer moving along the red vector arrow $v$ is processed with two different apodization functions: a conventional bell-shaped apodization function to obtain the axial velocity component $v_{z}$ (a), and a two-peak apodization function to obtain the transverse velocity component $v_{x}$ (b). The apodization functions in red are superimposed on the transducers, the active elements in transmit are colored blue, the emitted signal is represented by solid lines and the scattered signal is represented by dotted lines (Hansen et al. 2016a). Reprinted with permission from Ultrasound in Medicine and Biology.

Each scan sequence of $14 \mathrm{~s}$ of recording corresponded to 225 frames. Direction and velocity of blood flow were given by the color map and indicated by the superimposed vector arrows (Fig. 2). On the US scanner, vector velocity estimates were displayed in real time, but without any quantification of velocities available. Thus, scan sequences were analyzed off-line using MATLAB (The MathWorks, Natick, MA, USA), as previously described (Pedersen et al. 2012).
Scan sequences in SAX view with low and high PRF settings were visually inspected for helical motion, and all frames with clear helical motion were analyzed. By use of streamlines for displaying blood motion, the center of the helical flow was recognized, as illustrated in Figure 3. A grid defining sub-areas in the 2-D vector field was set, so the central helical motion was encompassed in a sub-area. For each sub-area, the curl of the $z$-component was calculated as

Table 1. Averaged vector flow imaging parameters by patient group

\begin{tabular}{|c|c|c|c|c|c|c|}
\hline Group & View & $\mathrm{PRF}(\mathrm{kHz})$ & $V_{\max }(\mathrm{cm} / \mathrm{s})$ & Gain $(\%)$ & Wall filtering $(\mathrm{Hz})$ & $\begin{array}{l}\text { Depth in B-mode } \\
\text { image }(\mathrm{cm})\end{array}$ \\
\hline \multirow[t]{4}{*}{ I } & LAX & $10.5(1.5)$ & $314.4(44.3)$ & $54.8(5.5)$ & 523.7 (73.7) & $4.9(0.4)$ \\
\hline & SAX & & & & & \\
\hline & Diastole & $1.3(0.4)$ & $38.1(10.8)$ & 51.9 (12.9) & $63.5(17.9)$ & $4.9(0.4)$ \\
\hline & Systole & $5.0(0.6)$ & $143.9(14.8)$ & $54.9(7.0)$ & 239.7 (24.6) & $4.9(0.4)$ \\
\hline \multirow[t]{4}{*}{ IIa } & LAX & $10.8(0.8)$ & $323.9(23.0)$ & $52.7(3.0)$ & 539.5 (38.4) & $5.0(0.2)$ \\
\hline & SAX & & & & & \\
\hline & Diastole & $2.3(0.6)$ & $68.2(18.1)$ & 53.9 (3.9) & $113.7(30.3)$ & $5.0(0.2)$ \\
\hline & Systole & $10.2(1.5)$ & $305.5(43.9)$ & $54.0(5.0)$ & $508.9(73.2)$ & $5.0(0.2)$ \\
\hline \multirow[t]{4}{*}{ IIb } & LAX & $10.3(1.0)$ & 309.7 (28.9) & $52.1(3.8)$ & $515.9(48.2)$ & $5.0(0.2)$ \\
\hline & SAX & & & & & \\
\hline & Diastole & $2.2(0.7)$ & $65.9(20.4)$ & $53.0(3.4)$ & $109.9(34.1)$ & $5.0(0.2)$ \\
\hline & Systole & $9.5(1.4)$ & $285.7(41.4)$ & $53.0(3.4)$ & $475.9(69.0)$ & $5.0(0.2)$ \\
\hline
\end{tabular}

LAX $=$ long-axis; SAX $=$ short-axis.

Values are the mean or frequency (standard deviation). 

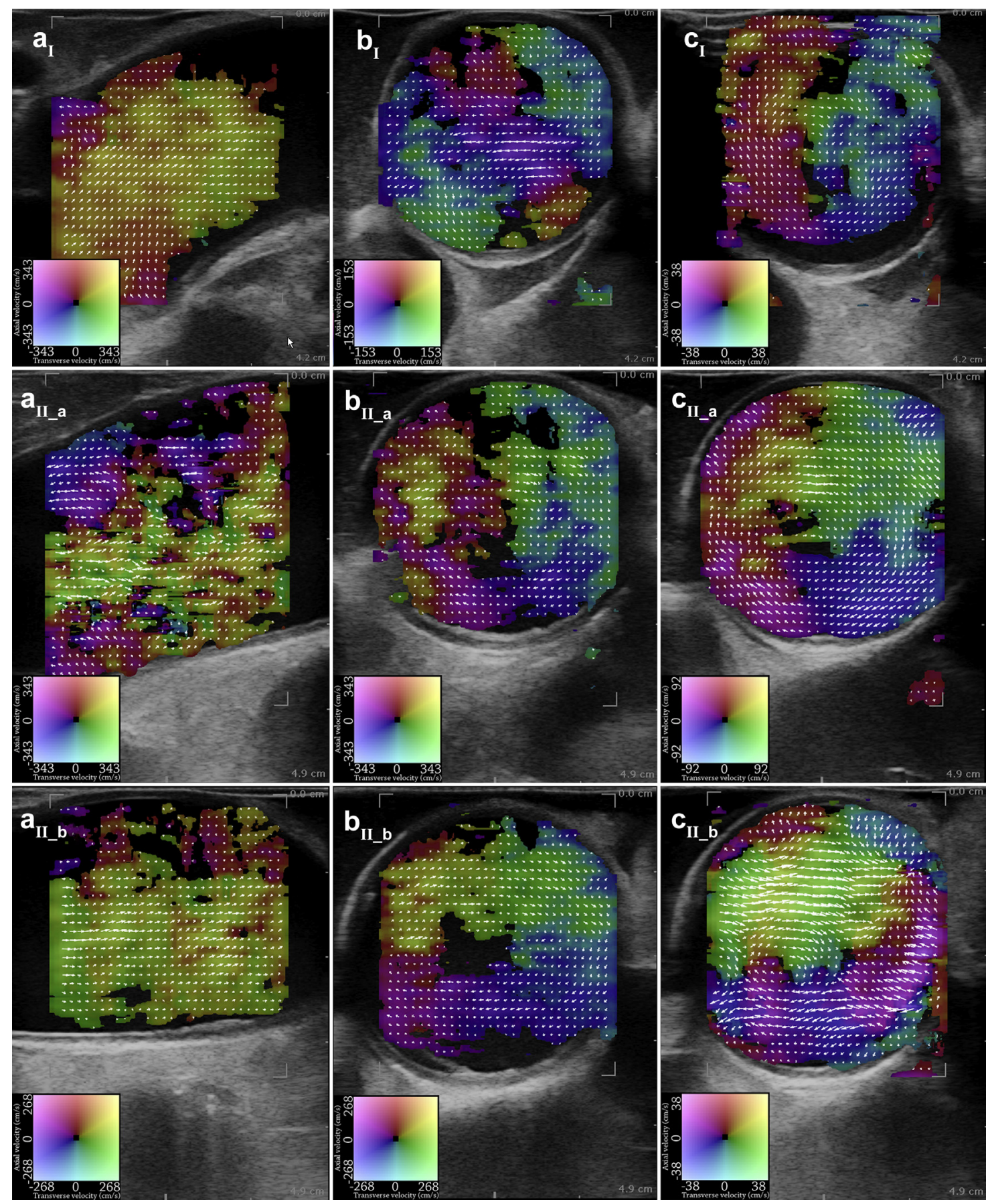

Fig. 2. Scans of one patient with a normal aortic valve (group I) and one patient with aortic valve stenosis before (group IIa) and after (group IIb) valve replacement. (a) Recordings in long-axis view during systole. (b,c) Recordings in shortaxis view during systole (b) and diastole (c). The flow angle of the vector velocity correlates to the pixel color, whereas the flow magnitude correlates to pixel intensity as indicated by the color map and the superimposed vector arrows. Patient group is denoted by the subscript.

$$
(\nabla \times V)_{z}=\frac{\partial}{\partial x} V_{y}-\frac{\partial}{\partial y} V_{x}
$$

where $(\nabla \times V)_{z}$ is the $z$-component of the curl in a 2-D vector field $v$ composed of two vector velocity components $\left[V_{x}, V_{y}\right]$, as described by Lautrup (2005). The vector velocity components $\left[V_{x}, V_{y}\right]$ corresponded to the axial velocity component $v_{z}$ and the transverse velocity component $v_{x}$ obtained by VFI. This provided the rotational frequency in rotations per second, where 

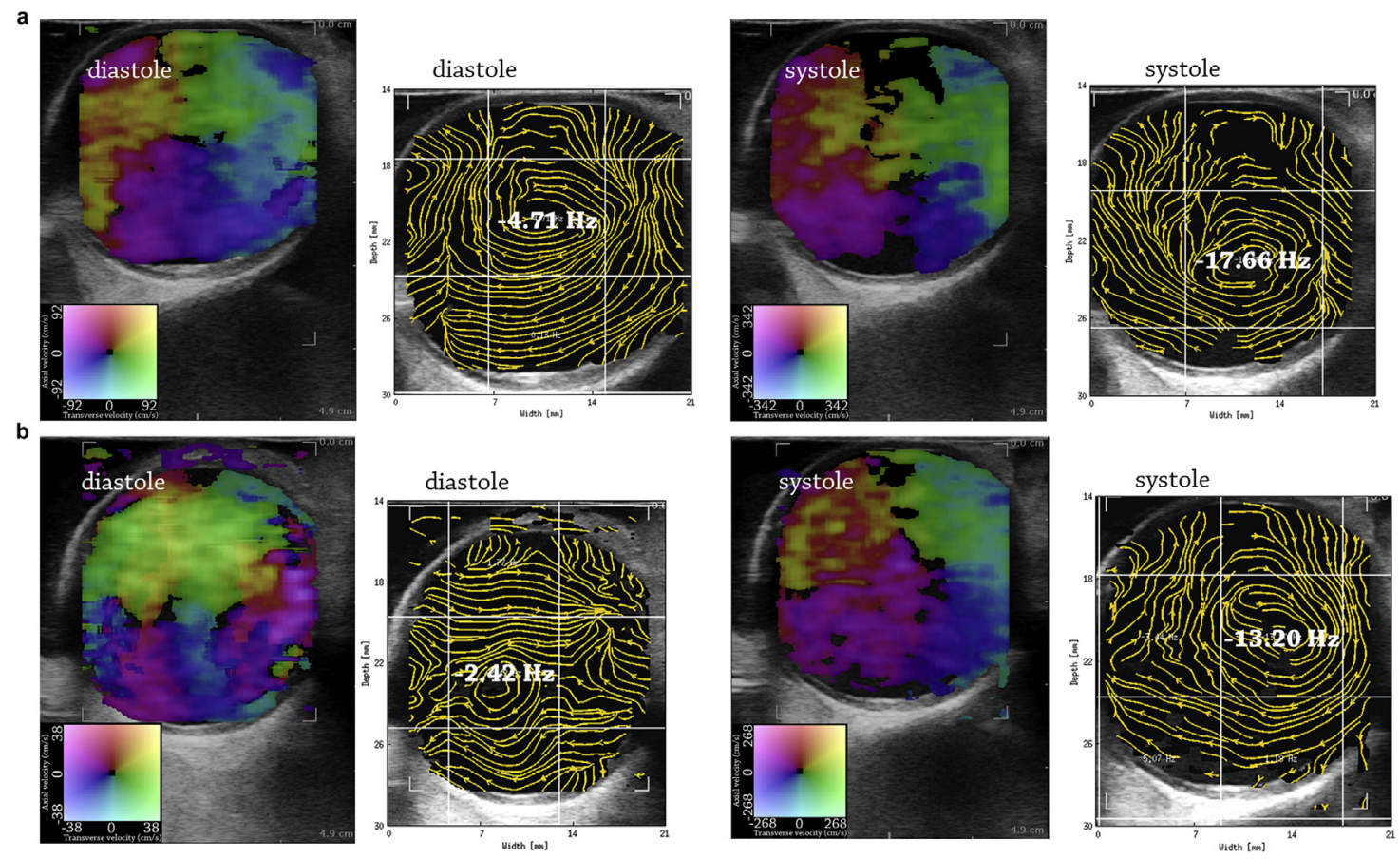

Fig. 3. Secondary helical flow obtained in short-axis view during diastole and systole for one patient from group II before (a) and after (b) aortic valve replacement. For each vector velocity map, the corresponding streamlines with rotational frequency calculated for sub-areas using eqn (1) are shown. Sub-areas are indicated by the superimposed grid, and the rotational frequency of the central sub-area is highlighted for each frame. A negative rotational frequency corresponds to a clockwise rotation. Diastole and systole are denoted by the subscripts.

the sign indicated the rotational direction; that is, a negative sign corresponded to a clockwise rotation and a positive sign to a counterclockwise rotation. The central sub-area had the highest frequencies, and the averaged frequency along with the rotational direction was reported for each patient (Fig. 3).

In LAX view, the primary blood flow during systole was examined with vector concentration, corresponding to a calculation of vector angle diversity within a ROI, that is, a measure of flow complexity. The vector concentration was found as follows. For each position $i$ in the vector map, where the axial velocity component $v_{z}$ and the transverse velocity component $v_{x}$ were estimated, the flow angle $\theta_{i}$ of the vector was calculated as

$$
\theta_{i}=\arctan \left(v_{z, i}, v_{x, i}\right)
$$

Each flow angle $\theta_{i}$ was represented on the unit circle as $p_{i}=\left(x_{i}, y_{i}\right)$, where $x_{i}=\cos \left(\theta_{i}\right)$ and $y_{i}=\sin \left(\theta_{i}\right)$. For each ROI encompassing the entire vessel, the mean values for $x_{i}$ and $y_{i}$ were found with

$$
\bar{x}=\frac{1}{n} \sum_{i=1}^{n} \cos \left(\theta_{i}\right)
$$

$$
\bar{y}=\frac{1}{n} \sum_{i=1}^{n} \sin \left(\theta_{i}\right)
$$

To quantify flow complexity, the vector concentration $r$ was found by using Pythagoras' theorem

$$
r=\sqrt{\bar{x}^{2}+\bar{y}^{2}}
$$

where $r$ is 1 for perfect laminar flow, and decreases toward zero with increasing complex flow (Fig. 4). Hence, for a chaotic flow, the vectors pointed in all random directions, and their average was, thus, close to zero, whereas the opposite was found for laminar flow, where the average was going toward 1, corresponding to the radius of the unit circle. The vector concentration $r$ of the blood flow during peak systole in the ascending aorta was found for each patient as an average of three consecutive systoles taken from the beginning of the examined scan sequence.

Thus, vector velocities recorded in SAX view were used for quantifying helical motion of the secondary flow using eqn (1), whereas vector velocities recorded in LAX view were used for quantifying flow complexity of the primary flow using eqns (2)-(5). Analyses of 

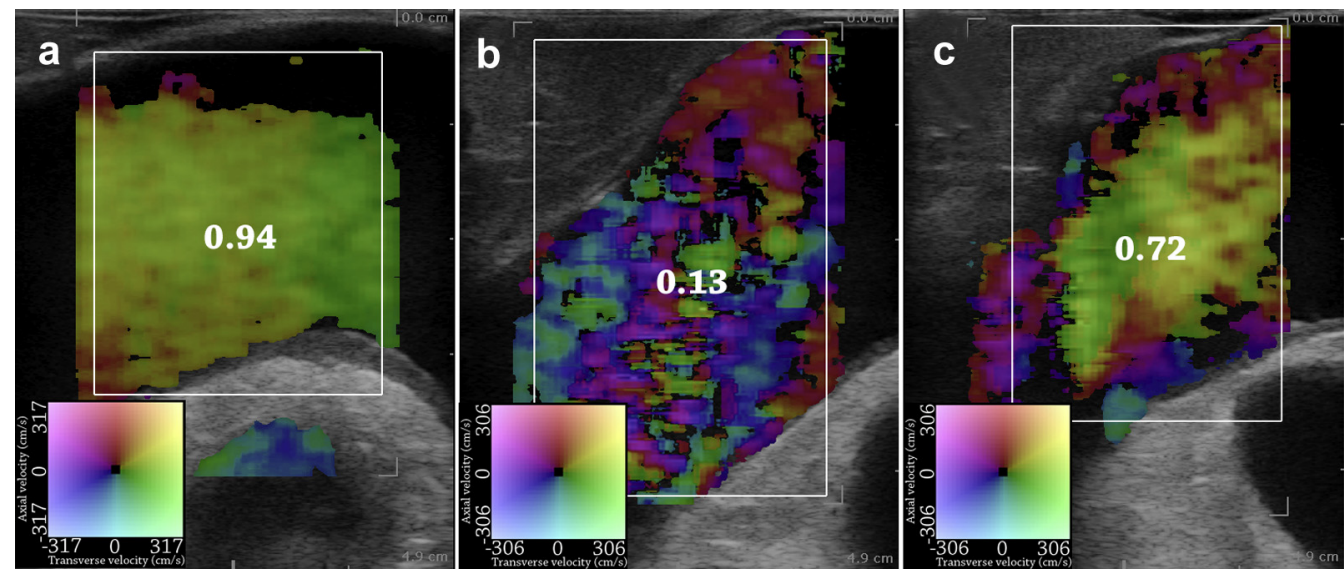

Fig. 4. Systolic flow obtained in long-axis view for two patients. (a) Recording of a patient from group I. (b,c) Recordings from a patient in group II before (b) and after (c) aortic valve replacement. The vector concentration is calculated from the vector estimates within the region of interest using eqns (2)-(5).

helical motion and vector concentration were performed blinded to the group status of the patients by K.L.H.

\section{Transesophageal echocardiography}

A Philips iE33 scanner equipped with a Philips X72 t transducer (Philips Healthcare, Eindhoven, The Netherlands) using continuous wave US was used for TEE. Scan parameters were adjusted for flow imaging, and scan sequences were obtained through the aortic valve aperture in the deep transgastric LAX view, with the line of interrogation placed along the left ventricular outlet tract and the ascending aorta. Peak systolic velocity was reported for each patient as an average of three heart cycles and was obtained just before the VFI examination. Thus, for patients with aortic valve stenosis, peak systolic velocity was measured twice, before and after aortic valve replacement.

\section{STATISTICS}

Data were initially analyzed with the appropriate descriptive statistics (means and standard deviations for numerical outcomes and frequencies for categorical outcomes). Comparisons of continuous variables, that is, vector concentration, rotational frequency and peak systolic velocity, were calculated with paired and unpaired Student $t$-tests, whereas comparisons of categorical variables, that is, direction of rotation and number of vortices, were calculated with Fisher's exact test for paired data and McNemar's test for unpaired data. Associations between continuous variables, that is, peak systolic velocities versus vector concentration and rotational frequency, were tested with Pearson's correlation, and comparisons of continuous and categorical variables, that is, peak systolic velocity versus direction of rotation and number of vortices, were calculated with one-way analysis of variance (ANOVA) (Altman 1991). To account for chance findings caused by multiple comparisons, the Bonferroni correction was employed. Accordingly, a $p$ value $<0.05$ after Bonferroni correction was considered to indicate significance. Statistical analyses were performed with SPSS Statistics, Version19 (IBM, Armonk, NY, USA).

\section{RESULTS}

In Table 2 are the results of the descriptive statistics on the data and of the statistical tests of the comparisons. Analyses with Pearson's correlation on pooled data from patient groups I and IIa revealed that TEE-derived peak systolic velocities were significantly associated with vector concentration $(p<0.0001, R=0.88)$ and rotational frequency of helical flow during diastole $(p<0.0001, R=0.87)$ and systole $(p<0.0001$, $R=0.94)$. Analyses with one-way ANOVA on pooled data from groups I and IIa indicated that TEE-derived peak systolic velocities were not associated with either rotational direction of the helical flow (diastole: $p=0.65$, systole: $p=0.03$ ) or number of vortices (diastole: $p=0.09$, systole: $p=0.11$ ). Scatterplots of vector concentration during systole and rotational frequency of the helical flow during systole and diastole correlated to peak systolic velocity are provided in Figure 5.

\section{DISCUSSION}

Previous VFI studies of blood flow in the ascending aorta reported that secondary flow was present in all patients examined, though no correlation to aortic valve stenosis was found (Hansen et al. 2015, 2016a, 2016b). As in other studies with TEE, MRI and computational fluid dynamics, the previous VFI studies were concerned 
Table 2. Descriptive statistics and results of comparisons with $p$-values

\begin{tabular}{|c|c|c|c|c|c|c|}
\hline \multirow[b]{2}{*}{ Group } & \multicolumn{3}{|c|}{ Data } & \multicolumn{3}{|c|}{$p$ Value } \\
\hline & I & IIa & $\mathrm{IIb}$ & I vs. IIa & I vs. IIb & IIa vs. IIb \\
\hline Vector concentration & $0.79(0.09)$ & $0.33(0.12)$ & $0.61(0.09)$ & $<0.0001^{*} \mathrm{~B}$ & $<0.0001 * \mathrm{~B}$ & $<0.0001 * \mathrm{~A}$ \\
\hline \multicolumn{7}{|l|}{ Rotation $(\mathrm{Hz})$} \\
\hline Diastole & $1.64(0.42)$ & $4.14(1.16)$ & $3.85(1.05)$ & $<0.0001 * \mathrm{~B}$ & $<0.0001 * \mathrm{~B}$ & $0.47 \mathrm{~A}$ \\
\hline Systole & $4.37(1.71)$ & $17.33(2.30)$ & $12.48(2.12)$ & $<0.0001 * \mathrm{~B}$ & $<0.0001 * \mathrm{~B}$ & $0.001 * \mathrm{~A}$ \\
\hline \multicolumn{7}{|l|}{ Direction of rotation } \\
\hline Diastole & & & & $0.63 \mathrm{C}$ & $0.30 \mathrm{C}$ & $1.0 \mathrm{D}$ \\
\hline CW & 6 & 8 & 9 & & & \\
\hline $\mathrm{CCW}$ & 4 & 2 & 1 & & & \\
\hline Systole & & & & $0.02 \mathrm{C}$ & $0.005^{*} \mathrm{C}$ & $1.0 \mathrm{D}$ \\
\hline CW & 2 & 8 & 9 & & & \\
\hline $\mathrm{CCW}$ & 8 & 2 & 1 & & & \\
\hline \multicolumn{7}{|l|}{ Number of vortices } \\
\hline Diastole & & & & $0.21 \mathrm{C}$ & $0.21 \mathrm{C}$ & $1.0 \mathrm{D}$ \\
\hline 1 & 7 & 10 & 10 & & & \\
\hline 2 & 3 & 0 & 0 & & & \\
\hline Systole & & & & $0.06 \mathrm{C}$ & $0.06 \mathrm{C}$ & $1.0 \mathrm{D}$ \\
\hline 1 & 4 & 9 & 9 & & & \\
\hline 2 & 6 & 1 & 1 & & & \\
\hline Peak systolic velocity $(\mathrm{cm} / \mathrm{s})$ & $124.0(20.7)$ & $346.1(75.9)$ & $232.4(37.4)$ & $<0.0001 * \mathrm{~B}$ & $<0.0001 * \mathrm{~B}$ & $0.002 * \mathrm{~A}$ \\
\hline
\end{tabular}

$\mathrm{CW}=$ clockwise $\mathrm{CCW}=$ counterclockwise; $\mathrm{PRF}=$ pulse repetition frequency.

* Significant association after Bonferroni correction. A-D are the tests used for comparisons: A = paired Student t-test; B = unpaired Student t-test; $\mathrm{C}=$ Fisher's exact test; $\mathrm{D}=$ McNemar's test. For patients with Dean flow, the direction of the most prominent vortex was used.

with secondary helical flow during late systole and diastole, thus omitting the peak systolic rotational component (Bogren and Buonocore 1999; Koh et al. 2001; Morbiducci et al. 2009, 2011; Tse et al. 2012). However, two recent studies using VFI reported that a secondary rotational flow was present during peak systole as a short burst of much faster rotation, though severely obscured because of aliasing (Hansen et al. 2016a, 2016b). The systolic rotational component has been addressed in other studies; for example, Tse et al. (2012) found helical flow during systole using computational fluid dynamics, and von Spiczak et al. (2015) reported that helical flow was most pronounced during midsystole in healthy volunteers with reduced vorticity during diastole, which in the present study likewise was found for patients with normal, stenotic and replaced aortic valves.

This is the first study indicating that peak systolic velocity and aortic valve stenosis are associated with the frequency of the secondary rotation during systole. This is not surprising, though not previously reported, as the systolic blood flow in the ascending aorta, apart from the torsion of the ascending aorta, is shaped by the passage through the aortic valve. Therefore, peak systolic velocities, and not diastolic velocities, are used for the grading of aortic valve stenosis (Nishimura et al. 2014). In the present study, however, the diastolic rotation also correlated to peak systolic velocities, in contrast to previous VFI studies, which could be explained by the separated data collection during diastole and systole with low and high PRF settings (Hansen et al. 2015, 2016b).
Two recent MRI studies have indicated an increased secondary flow in the ascending aorta in patients with aortic valve stenosis (von Knobelsdorff-Brenkenhoff et al. 2016) and in patients with implanted biological aortic prostheses (von Knobelsdorff-Brenkenhoff et al. 2014) compared with normal volunteers. Despite qualitative evaluations and no measurements before valve replacement, the findings are similar to the results of this study, in which both native stenotic valves and valve prostheses produced faster secondary rotations than normal aortic valves.

The secondary flow in the ascending aorta has been examined primarily in patients with bicuspid aortic valves. This condition seems to enhance the rotation just as aortic valve stenosis does (Hope et al. 2011; Meierhofer et al. 2013; Schnell et al. 2016). Hope et al. (2011) stated that helical flow is partly governed by the aortic valve, as a bicuspid valve produces more pronounced helical flow than normal tricuspid valves. The present study and a recent VFI study support Hope et al., as the speed and, to a lesser extent, the direction of the secondary rotation in the ascending aorta seem to be governed by the aortic valve (Hansen et al. 2016b). Normal and stenotic aortic valves produce different secondary flow motion, and for patients with stenotic and replaced valves, flow changes for the individual patient have likewise been observed, including directional change (Hansen et al. 2016b). Although aortic valve stenosis seems to promote a fast clockwise singular vortical formation, a large proportion $(60 \%)$ of patients with normal valves had a slower moving systolic Dean flow, 

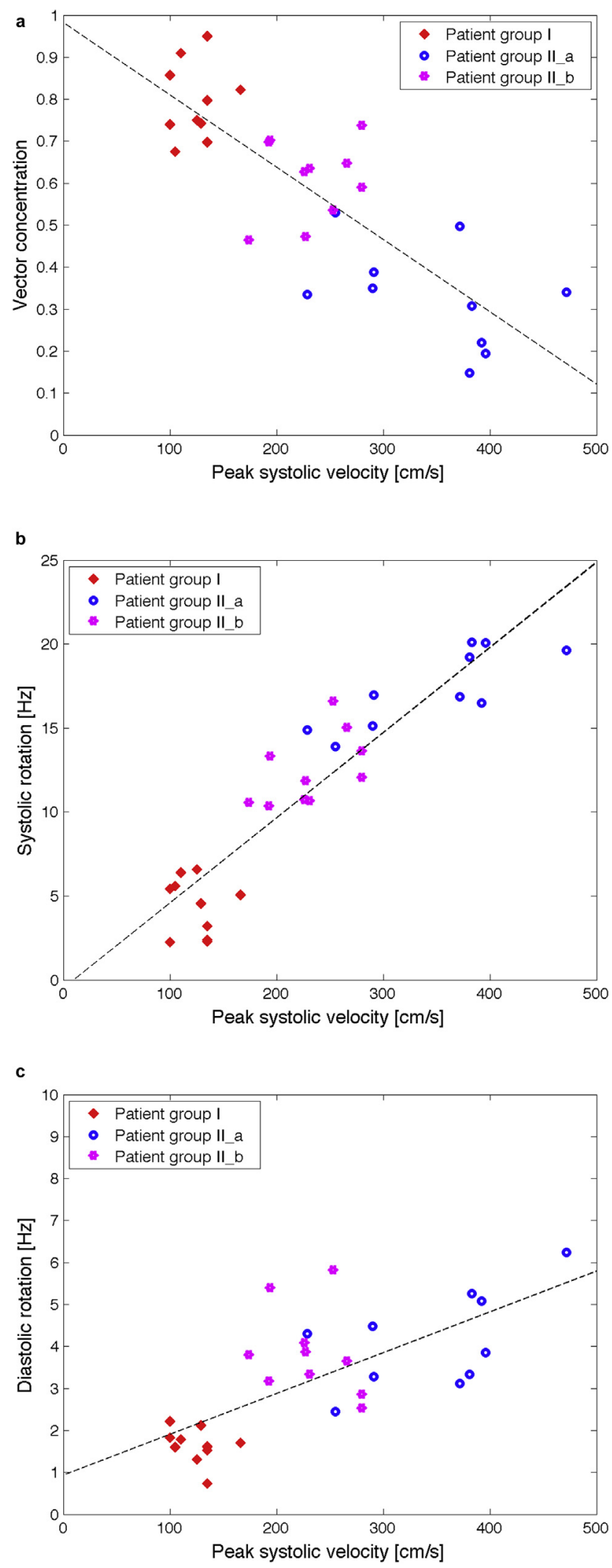

Fig. 5. Scatterplots of vector concentration during systole (a), rotational frequency of the helical flow during systole (b) and during diastole (c), correlated to peak systolic velocity. Line of best fit is represented by a dashed line in each plot. 
that is, two opposing vortices (Dean 1927), where the largest vortex primarily had counterclockwise flow direction. Using MRI, Hope et al. found Dean flow in the ascending aorta in $50 \%$ of a healthy study population, $80 \%$ of whom had counterclockwise rotation (Hope et al. 2007). In a previous VFI study, clockwise rotation was found primarily for normal aortic valves, and counterclockwise rotation, for stenotic and replaced aortic valves. Furthermore, Dean flow was found for $20 \%$ of normal and stenotic valves, but not for replaced aortic valves. This is in accordance with the present study, when taking into account that diastolic rotations were examined previously with VFI (Hansen et al. 2016b).

In SAX view, the out-of-plane flow going in the primary direction can be so strong that the rotational motion during systole will be missed, as the blood speckle will traverse the short-axis scan plane faster than the system can sample. Furthermore, as the frequency of the rotation according to this study has a linear relationship with peak systolic velocity of the primary flow, the rotation frequency can exceed the Nyquist limit in patients with severe aortic stenosis and very high peak systolic velocities. In both situations, that is, too strong primary or secondary flow, the result of the VFI assessment in SAX view during systole will be unreliable vector velocity estimates revealing a chaotic flow pattern. In this study, however, the systolic rotation was found for all patients, and no aliasing of secondary flow was observed.

Apart from the secondary helical flow in SAX view, the systolic blood flow in LAX view of the ascending aorta was investigated with vector concentration, a recently introduced parameter for flow characterization (Hansen et al. 2015, 2016b). As indicated in the previous studies, vector concentration was strongly correlated to peak systolic velocity and significantly different in the three groups. A linear relationship between vector concentration and peak systolic velocity was present, even for the highest peak systolic velocities, where aliasing was expected. As previously stated, aliased blood flow results in increased errors in the velocity estimate, but if the flow is laminar, it remains uniform when aliasing occurs, thus affecting the vector concentration estimate to a lesser extent (Hansen et al. 2015). However, some reduction in vector concentration has to be expected when aliasing occurs, even if the flow is laminar, as the central part of the flow with the highest and aliased velocities will appear retrograde, whereas the peripheral flow will remain antegrade. This will create at least two opposing flow angles within the ROI and result in increasing flow complexity. Nevertheless, this effect is, according to the initial results, less prominent than the effect of the actual increase in flow complexity for increasing aortic valve stenosis as illustrated in Figure 5. Therefore, the vector concentration achieved with vector flow estimation is considered a PRF-independent in vivo measure of flow complexity corresponding to the Reynolds number (Reynolds 1883) and correlating to peak systolic velocity and aortic valve stenosis (Hansen et al. 2016b).

As VFI is based on pulsed ultrasound emission, the vector concentration has an advantage to velocity estimation using continuous wave ultrasound, as data sampling is done at a specific depth and not along a line. Moreover, vector concentration using flow data of the full vector map is less dependent on capturing the highest velocities for flow evaluation and, thus, less hampered by shadowing calcifications and eccentric flow, which often are present in the ascending aorta along with aortic valve stenosis (Hansen et al. 2016b; Sigovan et al. 2015; Weisenberg et al. 2005). With VFI, a method for evaluation of complex flow patterns without the angle dependency found in conventional Doppler ultrasound is introduced.

Magnetic resonance imaging has a spatial resolution of $2 \mathrm{~mm}$, and records vector estimates from an averaged cardiac cycle using electrocardiographic gating over a long acquisition time (Markl et al. 2011), whereas VFI is a real-time method with a spatial isotropic resolution of approximately $1 \mathrm{~mm}$ (Udesen and Jensen 2006). The peak systolic rotation takes place in a short time, which can be difficult to capture in MRI, where averaging is necessary. Meanwhile, the rotation during the diastole temporally covers more of the heart cycle and, thus, is easier to align and depict, when performing averaging over several heart cycles. By observing the VFI sequences in SAX view, it was clear that the vortices changed position from cycle to cycle, which potentially can hide vortex formation in the MRI evaluation. Furthermore, just as VFI estimation is dependent on a correctly adjusted PRF, MRI estimation is dependent on velocity encoding, which, when incorrectly set, can obscure flow patterns (Callaghan et al. 2015; Hope et al. 2007).

Assessment of blood flow in the ascending aorta with VFI is limited to an epi-aortic scan approach because of the penetration depth limited by the transducer setup. However, implementations of VFI on phased and curved array transducers with increased penetration depth have been accomplished (Jensen et al. 2015; Pihl et al. 2012).

Assessment of the complex blood flow in the ascending aorta is improved with vector flow imaging compared with conventional Doppler US, but as only 2-D flow is obtained in-plane, the out-of-plane flow patterns are missed. This has been reported to be an important limitation in volume flow estimation of the ascending aorta using VFI (Hansen et al. 2017), and is probably also a limitation in the assessment of flow complexity. However, 3-D vector flow using VFI has been achieved, and preliminary results obtained in vivo 
have recently been published proving that VFI in 3-D is feasible (Holbek et al. 2017).

The frequency of the secondary rotation increased in patients with aortic stenosis; however, whether this is beneficial or injurious to the patient remains to be answered in future studies with VFI. Furthermore, the systolic rotation in the ascending aorta should also be investigated in relation to development of aortic aneurysm in patients with aortic valve stenosis and in patients with bicuspid aortic valve to examine possible associations (Meierhofer et al. 2013). Finally, VFI studies of complex flow changes in valve replacement with mechanical and biologic prostheses should be assessed, and vector concentration should be applied to assessment of stenosis in other vessel segments, for example, the carotid and femoral arteries, and in pediatrics for evaluation of congenital heart conditions.

\section{CONCLUSIONS}

Cardiac VFI is a promising technique that can provide new insight into cardiac flow with the use of new flow parameters and insonation windows. This study has revealed that the secondary helical flow in the ascending aorta consists of systolic and diastolic components, both of which are associated with the peak systolic flow. Furthermore, the complexity of the primary blood flow during systole evaluated with the VFI parameter vector concentration has, as in a previous study, exhibited a strong association with peak systolic flow. Thus, this study using intra-operative cardiac VFI indicates that both helical flow and flow complexity are related to aortic valve stenosis.

Acknowledgments-The study was supported by Grant 82-2012-4 from The Danish National Advanced Technology Foundation and by BK Medical ApS.

\section{REFERENCES}

Altman DG. Practical statistics for medical research. London: Chapmann \& Hall/CRC; 1991.

American Society of Anesthesiologists and Society of Cardiovascular Anesthesiologists Task Force on Transesophageal Echocardiography. Practice guidelines for perioperative transesophageal echocardiography: An updated report by the American Society of Anesthesiologists and the Society of Cardiovascular Anesthesiologists Task Force on Transesophageal Echocardiography. Anesthesiology 2010;112:1084-1096.

Bogren HG, Buonocore MH. 4-D magnetic resonance velocity mapping of blood flow patterns in the aorta in young vs. elderly normal subjects. J Magn Reson Imaging 1999;10:861-869.

Callaghan FM, Kozor R, Sherrah AG, Vallely M, Celermajer D, Figtree GA, Grieve SM. Use of multi-velocity encoding 4-D flow MRI to improve quantification of flow patterns in the aorta. J Magn Reson Imaging 2015;43:352-363.

Cloutier G, Allard L, Durand LG. Changes in ultrasonic Doppler backscattered power downstream of concentric and eccentric stenoses under pulsatile flow. Ultrasound Med Biol 1995;21:59-70.

Dean WR. Note on the motion of fluid in a curved pipe. Philos Mag 1927:4:208-223.
Elbaz MS, Calkoen EE, Westenberg JJ, Lelieveldt BP, Roest AA, van der Geest RJ. Vortex flow during early and late left ventricular filling in normal subjects: Quantitative characterization using retrospectively-gated 4-D flow cardiovascular magnetic resonance and three-dimensional vortex core analysis. J Cardiovasc Magn Reson $2014 ; 16: 78$.

Frazin LJ, Vonesh MJ, Chandran KB, Shipkowitz T, Yaacoub AS, McPherson DD. Confirmation and initial documentation of thoracic and abdominal aortic helical flow. An ultrasound study. ASAIO J 1996:42:951-956.

Hansen KL, Moller-Sorensen H, Pedersen MM, Hansen PM, Kjaergaard J, Lund JT, Nilsson JC, Jensen JA, Nielsen MB. First report on intraoperative vector flow imaging of the heart among patients with healthy and diseased aortic valves. Ultrasonics 2015;56: 243-250.

Hansen KL, Moller-Sorensen H, Kjaergaard J, Jensen MB, Lund JT, Pedersen MM, Lange T, Jensen JA, Nielsen MB. Analysis of systolic backflow and secondary helical blood flow in the ascending aorta using vector flow imaging. Ultrasound Med Biol 2016a;42:899-908.

Hansen KL, Moller-Sorensen H, Kjaergaard J, Jensen MB, Lund JT, Pedersen MM, Lange T, Jensen JA, Nielsen MB. Intra-operative vector flow imaging using ultrasound of the ascending aorta among 40 patients with normal, stenotic and replaced aortic valves. Ultrasound Med Biol 2016b;42:2414-2422.

Hansen KL, Moller-Sorensen H, Kjaergaard J, Jensen MB, Lund JT, Pedersen MM, Olesen JB, Jensen JA, Nielsen MB. Vector flow imaging compared with conventional Doppler ultrasound and thermodilution for estimation of blood flow in the ascending aorta. Ultrason Imaging 2017:39:3-18.

Holbek S, Ewertsen C, Bouzari H, Pihl MJ, Hansen KL, Stuart MB, Thomsen C, Nielsen MB, Jensen JA. Ultrasonic 3-D vector flow method for quantitative in vivo peak velocity and flow rate estimation. IEEE Trans Ultrason Ferroelectr Freq Control 2017;64: 544-554.

Hope TA, Markl M, Wigstrom L, Alley MT, Miller DC, Herfkens RJ. Comparison of flow patterns in ascending aortic aneurysms and volunteers using four-dimensional magnetic resonance velocity mapping. J Magn Reson Imaging 2007;26:1471-1479.

Hope MD, Hope TA, Meadows AK, Ordovas KG, Urbania TH, Alley MT, Higgins CB. Bicuspid aortic valve: Four-dimensional MR evaluation of ascending aortic systolic flow patterns. Radiology 2010;255:53-61.

Hope MD, Hope TA, Crook SE, Ordovas KG, Urbania TH, Alley MT, Higgins CB. 4-D flow CMR in assessment of valve-related ascending aortic disease. JACC Cardiovasc Imaging 2011;4: $781-787$.

Hutchison SJ, Rosin BL, Curry S, Chandraratna PA. Transesophageal echocardiographic assessment of lesions of the right ventricular outflow tract and pulmonic valve. Echocardiography 1996;13: 21-34.

Jensen JA. A new estimator for vector velocity estimation. IEEE Trans Ultrason Ferroelect Freq Control 2001;48:886-894.

Jensen JA, Munk P. A new method for estimation of velocity vectors. IEEE Trans Ultrason Ferroelect Freq Control 1998;45:837-851.

Jensen JA, Brandt AH, Nielsen MB. Convex array vector velocity imaging using transverse oscillation and its optimization. IEEE Trans U1trason Ferroelect Freq Control 2015;62:2043-2053.

Kilner PJ, Yang GZ, Mohiaddin RH, Firmin DN, Longmore DB. Helical and retrograde secondary flow patterns in the aortic arch studied by three-directional magnetic resonance velocity mapping. Circulation 1993;88:2235-2247.

Koh TW, Parker KH, Kon M, Pepper JR. Changes in aortic rotational flow during cardiopulmonary bypass studied by transesophageal echocardiography and magnetic resonance velocity imaging: A potential mechanism for atheroembolism during cardiopulmonary bypass. Heart Vessels 2001;16:1-8.

Lautrup B. Physics of continuous matter: Exotic and everyday phenomena in the macroscopic world. Bristol: Institute of Physics; 2005.

Liu X, Fan Y, Deng X. Effect of spiral flow on the transport of oxygen in the aorta: A numerical study. Ann Biomed Eng 2009a;38:917-926.

Liu X, Pu F, Fan Y, Deng X, Li D, Li S. A numerical study on the flow of blood and the transport of LDL in the human aorta: The 
physiological significance of the helical flow in the aortic arch. Am J Physiol Heart Circ Physiol 2009b;297:H163-H170.

Liu X, Sun A, Fan Y, Deng X. Physiological significance of helical flow in the arterial system and its potential clinical applications. Ann Biomed Eng 2015;43:3-15.

Lurie F, Kistner RL. On the existence of helical flow in veins of the lower extremities. J Vasc Surg Venous Lymphat Disord 2013;1: $134-138$.

Markl M, Draney MT, Hope MD, Levin JM, Chan FP, Alley MT, Pelc NJ, Herfkens RJ. Time-resolved 3-dimensional velocity mapping in the thoracic aorta: Visualization of 3-directional blood flow patterns in healthy volunteers and patients. J Comput Assist Tomogr 2004;28:459-468.

Markl M, Kilner PJ, Ebbers T. Comprehensive 4-D velocity mapping of the heart and great vessels by cardiovascular magnetic resonance. J Cardiovasc Magn Reson 2011;13:7.

Meierhofer C, Schneider EP, Lyko C, Hutter A, Martinoff S, Markl M, Hager A, Hess J, Stern H, Fratz S. Wall shear stress and flow patterns in the ascending aorta in patients with bicuspid aortic valves differ significantly from tricuspid aortic valves: A prospective study. Eur Heart J Cardiovasc Imaging 2013;14:797-804.

Morbiducci U, Ponzini R, Rizzo G, Cadioli M, Esposito A, De Cobelli F, Del Maschio A, Montevecchi FM, Redaelli A. In vivo quantification of helical blood flow in human aorta by time-resolved three-dimensional cine phase contrast magnetic resonance imaging. Ann Biomed Eng 2009;37:516-531.

Morbiducci U, Ponzini R, Rizzo G, Cadioli M, Esposito A, Montevecchi FM, Redaelli A. Mechanistic insight into the physiological relevance of helical blood flow in the human aorta: An in vivo study. Biomech Model Mechanobiol 2011; 10:339-355.

Nishimura RA, Otto CM, Bonow RO, Carabello BA, Erwin JP 3rd, Guyton RA, O'Gara PT, Ruiz CE, Skubas NJ, Sorajja P, Sundt TM 3rd, Thomas JD, American College of Cardiology/ American Heart Association Task Force on Practice Guidelines. 2014 AHA/ACC guideline for the management of patients with valvular heart disease: Executive summary: A report of the American College of Cardiology/American Heart Association Task Force on Practice Guidelines. J Am Coll Cardiol 2014;63: $2438-2488$.

Pedersen MM, Pihl MJ, Hansen JM, Hansen PM, Haugaard P, Nielsen MB, Jensen JA. Arterial secondary blood flow patterns visualized with vector flow ultrasound. Proc IEEE Int Ultrason Symp 2011;1242-1245.

Pedersen MM, Pihl MJ, Haugaard P, Hansen JM, Hansen KL, Nielsen MB, Jensen JA. Comparison of real-time in vivo spectral and vector velocity estimation. Ultrasound Med Biol 2012;38: $145-151$.

Pedersen MM, Pihl MJ, Haugaard P, Hansen KL, Lange T, Lonn L, Nielsen MB, Jensen JA. Novel flow quantification of the carotid bulb and the common carotid artery with vector flow ultrasound. U1trasound Med Biol 2014;40:2700-2706.
Pihl MJ, Marcher J, Jensen JA. Phased-array vector velocity estimation using transverse oscillations. IEEE Trans Ultrason Ferroelect Freq Control 2012;59:2662-2675.

Reynolds O. An experimental investigation of the circumstances which determine whether the motion of water shall be direct or sinuous and of the law of resistance in parallel channels. Philos Trans R Soc Lond 1883;174:935-987.

Sarrami-Foroushani A, Nasr Esfahany M, Nasiraei Moghaddam A, Saligheh Rad H, Firouznia K, Shakiba M, Ghanaati H, Wilkinson ID, Frangi AF. Velocity measurement in carotid artery: Quantitative comparison of time-resolved 3-D phase-contrast MRI and imagebased computational fluid dynamics. Iran J Radiol 2015;12:e18286.

Schnell S, Smith DA, Barker AJ, Entezari P, Honarmand AR, Carr ML, Malaisrie SC, McCarthy PM, Collins J, Carr JC, Markl M. Altered aortic shape in bicuspid aortic valve relatives influences blood flow patterns. Eur Heart J Cardiovasc Imaging 2016;17:1239-1247.

Segadal L, Matre K. Blood velocity distribution in the human ascending aorta. Circulation 1987;76:90-100.

Sigovan M, Dyverfeldt P, Wrenn J, Tseng EE, Saloner D, Hope MD. Extended 3-D approach for quantification of abnormal ascending aortic flow. Magn Reson Imaging 2015;33:695-700.

Simpson IA, Sahn DJ, Valdes-Cruz LM, Chung KJ, Sherman FS, Swensson RE. Color Doppler flow mapping in patients with coarctation of the aorta: New observations and improved evaluation with color flow diameter and proximal acceleration as predictors of severity. Circulation 1988;77:736-744.

Stonebridge PA, Brophy CM. Spiral laminar flow in arteries? Lancet 1991;338:1360-1361.

Stringer DA, O'Halpin D, Daneman A, Liu P, Geary DF. Duplex Doppler sonography for renal artery stenosis in the post-transplant pediatric patient. Pediatr Radiol 1989;19:187-192.

Tse KM, Chang R, Lee HP, Lim SP, Venkatesh SK, Ho P. A computational fluid dynamics study on geometrical influence of the aorta on haemodynamics. Eur J Cardiothorac Surg 2012;43:829-838.

Udesen J, Jensen JA. Investigation of transverse oscillation method. IEEE Trans Ultrason Ferroelect Freq Control 2006;53:959-971.

von Knobelsdorff-Brenkenhoff F, Trauzeddel RF, Barker AJ, Gruettner H, Markl M, Schulz-Menger J. Blood flow characteristics in the ascending aorta after aortic valve replacement-A pilot study using 4 D-flow MRI. Int J Cardiol 2014:170:426-433.

von Knobelsdorff-Brenkenhoff F, Karunaharamoorthy A, Trauzeddel RF, Barker AJ, Blaszczyk E, Markl M, Schulz-Menger J. Evaluation of aortic blood flow and wall shear stress in aortic stenosis and its association with left ventricular remodeling. Circ Cardiovasc Imaging 2016;9:e004038.

von Spiczak J, Crelier G, Giese D, Kozerke S, Maintz D, Bunck AC. Quantitative analysis of vortical blood flow in the thoracic aorta using 4-D phase contrast MRI. PLoS One 2015;10:e0139025.

Weisenberg D, Sahar Y, Sahar G, Shapira Y, Iakobishvili Z, Vidne BA, Sagie A. Atherosclerosis of the aorta is common in patients with severe aortic stenosis: An intraoperative transesophageal echocardiographic study. J Thorac Cardiovasc Surg 2005;130:29-32. 